\title{
Is vitiligo associated with systemic aquaporin-3 deficiency?
}

\author{
Laxmisha Chandrashekar ${ }^{1}$, Medha Rajappa², Kalai Selvi Rajendiran $^{3}$, Malathi Munisamy $^{1}$ \\ ${ }^{1}$ Department of Dermatology, Jawaharlal Institute of Postgraduate Medical Education and Research, Puducherry, India \\ ${ }^{2}$ Department of Biochemistry, Jawaharlal Institute of Postgraduate Medical Education and Research, Puducherry, India \\ ${ }^{3}$ Department of Biochemistry, Panimalar Medical College Hospital and Research Institute, Chennai, India
}

Adv Dermatol Allergol 2021; XXXVIII (1): 156-158 DOI: https://doi.org/10.5114/ada.2021.104291

\begin{abstract}
Introduction: Recent studies on pathomechanisms of vitiligo have focused on the abnormality of keratinocytes that affect the melanocytes. Aquaporin-3 (AQP3) was implicated as a mechanism for keratinocyte apoptosis owing to the relationship between the PI3K/AKT pathway and the E-cadherin-catenin complex.

Aim: Based on this evidence, we undertook a cross-sectional study to assess the skin and blood AQP-3 levels in patients with non-segmental vitiligo in comparison to controls and to correlate these levels with malondialdehyde (MDA) levels and total antioxidant status (TAS) in the skin and blood of patients with non-segmental vitiligo and also with their disease activity.

Material and methods: Thirty-six patients with non-segmental vitiligo and 36 controls were included in this study. AQP3, TAS and MDA levels were assayed both in skin as well as in circulation.

Results: We observed that skin and plasma aquaporin and TAS were lowered and MDA levels were increased in patients with non-segmental vitiligo as compared to controls. There was a significant negative correlation of skin and plasma aquaporin levels with disease activity. We also observed the local and systemic AQP3 deficiency to correlate with the local and systemic oxidative stress in vitiligo.

Conclusions: Our results demonstrate a systemic and local AQP3 deficiency in vitiligo correlating with the disease severity and oxidative stress which might have therapeutic implications.
\end{abstract}

Key words: aquaporin-3, oxidative stress, vitiligo.

\section{Introduction}

Aquaporin-3 (AQP3), a member of the subfamily of aquaglyceroporins is expressed and localized in the basal and spinous layers of the epidermis regulating the epidermal structure and function. Recent studies on pathomechanisms of vitiligo have focused on the abnormality of keratinocytes that affect the melanocytes. Studies have shown that keratinocytes in the depigmented epidermis in patients with vitiligo were more vulnerable to apoptosis due to impaired phosphatidylinositol 3-kinase (PI3K)/serine/threonine protein kinase (Akt) activation resulting in reduced nuclear factor- $\kappa \mathrm{B}$ activation under increased tumour necrosis factor- $\alpha$ levels [1]. AQP3 was implicated as a mechanism for keratinocyte apoptosis owing to the relationship between the PI3K/AKT pathway and the E-cadherin-catenin complex. Reduced AQP3 levels associated with reductions in the levels of
E-cadherin, $\beta$ - and $\gamma$-catenins and PI3K were observed in keratinocytes from the depigmented epidermis of vitiligo [2]. In addition, delayed barrier recovery has been observed in vitiligo and is associated with a deficiency of $A Q P 3$ [3]. It has also been reported that $A Q P 3$ and unorthodox AQP8 mediate $\mathrm{H}_{2} \mathrm{O}_{2}$ uptake through mammalian cell membranes which can amplify or diminish downstream signalling cascades by acting as a physiological messenger, thereby implicating AQP3 deficiency in the systemic and local oxidative stress in vitiligo [4].

\section{Aim}

Based on this evidence, we undertook a cross-sectional study to assess the skin and blood AQP3 levels in patients with non-segmental vitiligo in comparison to controls and to correlate these levels with malondialdehyde (MDA) levels and total antioxidant status (TAS) in

Address for correspondence: Dr. Laxmisha Chandrashekar MD, DNB, Department of Dermatology, Jawaharlal Institute of Postgraduate Medical Education and Research (JIPMER), Dhanvantari Nagar, Puducherry 605006, India, phone: +91-98941-19058, fax: +91-413-2272067, e-mail: laxmishac@gmail.com Received: 21.01.2019, accepted: 21.05.2019. 
the skin and blood of patients with non-segmental vitiligo and also with their disease activity.

\section{Material and methods}

Thirty-six patients with non-segmental vitiligo and 36 age- and gender-matched healthy controls were included in this hospital-based, cross-sectional study, after obtaining clearance from the Institute Ethics Committee (Human Studies) (Approved as project no. JIP/ IEC/2013/1/111 dated 16 February 2013). The study protocol conformed to the ethical guidelines of the Declaration of Helsinki. Written informed consent was obtained from all study subjects, prior to participation in the study. Cases included patients with non-segmental vitiligo, attending the dermatology outpatient department of our institute. Controls were age- and gender-matched healthy volunteers. Cases and controls were excluded based on the following criteria: cases currently on therapy (local and systemic), inflammatory skin diseases, pregnancy, renal impairment and corneal inflammation. Five millilitres of venous blood was drawn from the antecubital vein of all study subjects and the plasma was separated and stored at $-80^{\circ} \mathrm{C}$ until analysis. Plasma was utilized for estimation of AQP3, MDA and TAS levels in circulation.

All patients with vitiligo were evaluated by a detailed history and clinical examination. The disease activity was assessed using the vitiligo disease activity index (VIDA) scoring [5]. Six mm punch biopsies were taken from lesional and non-lesional skin in 12 patients with non-segmental vitiligo. Control skin samples were taken from the skin of 12 patients undergoing split skin grafting, pinch grafts and punch grafts for management of various ulcers. Tissue homogenate was utilized for estimation of AQP3, MDA and TAS levels in the skin.

Plasma and tissue AQP3 levels were measured by an enzyme immunoassay (Cusabio Biotech Ltd., Wuhan, China) according to the manufacturer's instructions. Plasma and tissue TAS levels were assayed using a commercially available kit (Cayman Chemical Company, Ann Arbor, MI,
USA) according to the manufacturer's instructions. MDA levels in the skin and plasma were assayed by the method of Agarwal and Chase, using high-performance liquid chromatography (SPD-20A HPLC system, Shimadzu, Japan) [6]. All the assays were done in duplicate and the mean was taken to increase the reliability of the measurements.

\section{IRB Approval}

Approved as proposal no. JIP/IEC/2013/1/111 dated 16.02.2013 by the Institute Ethics Committee (Human Studies), JIPMER, Puducherry, India.

\section{Statistical analysis}

Statistical analysis was performed using IBM SPSS statistics version 20 for Windows. Baseline characteristics of cases and controls were analysed using descriptive statistics. The normality of continuous data was assessed by Kolmogorov-Smirnov test. The normally distributed data were described as mean \pm standard deviation and compared by independent student's $t$-test or paired t-test, as appropriate. Correlation analysis was done between the severity and activity of non-segmental vitiligo and levels of the biochemical parameters. All statistical analysis was carried out at $5 \%$ level of significance and $p$-value $<0.05$ was considered as significant.

\section{Results}

We observed that skin and plasma AQP3 and TAS were lowered and MDA levels were increased in patients with non-segmental vitiligo, as compared to controls (Table 1). There was also a significant difference in the tissue levels of study parameters between lesional and non-lesional skin of vitiligo patients (Table 2). There was a significant negative correlation of skin $(r=-0.745 ; p=0.005)$ and plasma $(r=-0.514 ; p=0.001)$ AQP3 levels with disease activity, as assessed by VIDA scoring. Similarly, skin $(r=0.776$, $p=0.003)$ and plasma $(r=0.822 ; p<0.0001)$ MDA levels and skin TAS $(r=-0.817 ; p=0.001)$ and plasma TAS $(r=-0.828 ; p<0.0001)$ correlated significantly with the

Table 1. Comparison of study parameters in plasma and skin between cases and controls

\begin{tabular}{|c|c|c|c|}
\hline Study parameters in plasma & $\begin{array}{l}\text { Non-segmental vitiligo } \\
\qquad(n=36)\end{array}$ & $\begin{array}{l}\text { Controls } \\
(n=36)\end{array}$ & $P$-value \\
\hline Plasma MDA [ $\mu \mathrm{mol} / \mathrm{l}]$ & $12.28 \pm 4.25$ & $7.50 \pm 1.52$ & $<0.0001$ \\
\hline Plasma TAS [mmol Trolox eq./l] & $2.56 \pm 1.14$ & $3.87 \pm 1.45$ & $<0.0001$ \\
\hline Plasma AQP-3 [ng/ml] & $13.63 \pm 4.39$ & $16.49 \pm 3.84$ & 0.005 \\
\hline Study parameters in skin & $\begin{array}{l}\text { Non-segmental vitiligo } \\
\qquad(n=12)\end{array}$ & $\begin{array}{l}\text { Controls } \\
(n=12)\end{array}$ & $P$-value \\
\hline Skin MDA [ $\mu \mathrm{mol} / \mathrm{g}$ w.w.] & $176.78 \pm 30.89$ & $134.36 \pm 22.51$ & 0.001 \\
\hline Skin TAS [mmol Trolox eq./g w.w.] & $5.75 \pm 2.09$ & $8.94 \pm 1.42$ & $<0.0001$ \\
\hline Skin AQP-3 [ng/g w.w.] & $7.08 \pm 1.93$ & $11.96 \pm 3.00$ & $<0.0001$ \\
\hline
\end{tabular}


Table 2. Comparison of study parameters in lesional and non-lesional skin in patients with non-segmental vitiligo

\begin{tabular}{lccc}
\hline Parameter & $\begin{array}{c}\text { Lesional skin } \\
(n=12)\end{array}$ & $\begin{array}{c}\text { Non-lesional skin } \\
(n=12)\end{array}$ & $P$-value \\
\hline MDA [ $\mu$ mol/g w.w.] & $176.78 \pm 30.89$ & $147.65 \pm 8.00$ & 0.005 \\
\hline TAS [mmol Trolox eq./g w.w.] & $5.75 \pm 2.09$ & $7.82 \pm 0.70$ & 0.004 \\
\hline AQP-3 [ng/g w.w.] & $7.08 \pm 1.93$ & $9.05 \pm 0.60$ & 0.003 \\
\hline
\end{tabular}

disease activity. There was a significant positive correlation between plasma and skin AQP3 levels with plasma and skin $\operatorname{TAS}(r=0.339, p=0.004 ; r=0.695 ; p<0.0001$, respectively), whilst there was a significant negative correlation between plasma and skin AQP3 levels with plasma and skin MDA levels $(r=-0.439, p<0.0001 ; r=-0.696, p<0.0001$, respectively).

\section{Discussion}

Hodeib et al. [7] in a recent study have shown a reduced immunohistochemical expression of AQP3 in vitiligo epidermis than in controls with an inverse correlation with VIDA, implicating AQP3 as a potential negative biological marker for vitiligo activity. In our study, in addition to reduction in tissue AQP3 levels in vitiligo we observed that plasma AQP3 levels were also reduced in vitiligo in comparison to controls and also correlating with disease severity suggesting a systemic AQP3 deficiency in vitiligo which has not been reported previously. Since AQP3 is known to mediate most of the glycerol movements across red cell membranes [8], RBC AQP3 could be implicated as the source of plasma AQP3 in our study. We also observed the local and systemic AQP3 deficiency to correlate with the local and systemic oxidative stress in vitiligo. Since AQP3 is involved in uptake of the physiological messenger $\mathrm{H}_{2} \mathrm{O}_{2}$ [4], its deficiency can result in oxidative stress and subsequent keratinocyte apoptosis thus implicating AQP3 deficiency in the pathogenesis of vitiligo. The AQP3 deficiency in vitiligo can also explain the lower risk of cutaneous malignancies in vitiliginous skin as AQP3 has been reported to be an important determinant in skin tumorigenesis [9].

\section{Conclusions}

The results of our study demonstrate systemic and local AQP3 deficiency in vitiligo correlating with disease severity and oxidative stress which might have therapeutic implications. However, further studies are warranted to identify the cause for local and systemic deficiency of AQP3 in vitiligo.

\section{Acknowledgments}

Funding for this research work as Indian Association of Dermatologists, Venereologists and Leprologists
(IADVL)-L'Oreal Hair and Skin Research Grant in the name of the corresponding author is gratefully acknowledged.

\section{Conflict of interest}

The authors declare no conflict of interest.

\section{References}

1. Kim NH, Lee AY. Reduced aquaporin3 expression and survival of keratinocytes in the depigmented epidermis of vitiligo. J Invest Dermatol 2010; 130: 2231-9.

2. Lee AY. Role of keratinocytes in the development of vitiligo. Ann Dermatol 2012; 24: 115-25.

3. Qin H, Zheng X, Zhong X, et al. Aquaporin-3 in keratinocytes and skin: its role and interaction with phospholipase D2. Arch Biochem Biophys 2011; 508: 138-43.

4. Miller EW, Dickinson BC, Chang CJ. Aquaporin-3 mediates hydrogen peroxide uptake to regulate downstream intracellular signaling. Proc Natl Acad Sci USA 2010; 107: 15681-6.

5. Njoo MD, Das PK, Bos JD, Westerhof W. Association of the Köbner phenomenon with disease activity and therapeutic responsiveness in vitiligo vulgaris. Arch Dermatol 1999; 135: 407-13.

6. Agarwal R, Chase SD. Rapid, fluorimetric-liquid chromatographic determination of malondialdehyde in biological samples. J Chromatogr B Analyt Technol Biomed Life Sci 2002; 775: 121-6.

7. Hodeib A, Hegab D, Rizk O, Mohammed S. Immunohistochemical expression of Aquaporin-3 protein in vitiligo: a new potential guide for disease activity. G Ital Dermatol Venereol 2017; 152: 348-54.

8. Roudier N, Verbavatz JM, Maurel C, et al. Evidence for the presence of aquaporin-3 in human red blood cells. J Biol Chem 1998; 273: 8407-12.

9. Hara-Chikuma M, Verkman AS. Prevention of skin tumorigenesis and impairment of epidermal cell proliferation by targeted Aquaporin-3 gene disruption. Mol Cell Biol 2008; 28: $326-32$. 